\title{
Functional magnetic resonance imaging study of word recognition in normal elders
}

\author{
Karen E. Anderson ${ }^{\mathrm{a}, \mathrm{d}, *}$, Gerard M. Perera ${ }^{\mathrm{c}}$, John Hilton ${ }^{\mathrm{b}, \mathrm{d}}$, Naomi Zubin ${ }^{\mathrm{b}, \mathrm{d}}$, \\ Robert Dela Paz ${ }^{\mathrm{c}}$, Yaakov Stern ${ }^{\mathrm{b}, \mathrm{d}}$ \\ ${ }^{a}$ Department of Neurology, Movement Disorders, University of Maryland School of Medicine, 22 South Greene Street, Baltimore, MD 21201, USA \\ ${ }^{\mathrm{b}}$ Gertrude H. Sergievsky Center, Department of Neurology, Columbia University, P\&S Box 16, 630 West 168th Street, New York, NY 10025, USA \\ ${ }^{\mathrm{c}}$ Department of Radiology, Columbia University, P\&S Box 16, 630 West 168th Street, New York, NY 10025, USA \\ ${ }^{\mathrm{d} C o g n i t i v e ~ N e u r o s c i e n c e ~ D i v i s i o n, ~ G e r t r u d e ~ H . ~ S e r g i e v s k y ~ C e n t e r, ~ C o l u m b i a ~ U n i v e r s i t y, ~ P \& S ~ B o x ~ 16, ~} 630$ West 168th Street, New York, NY 10025, USA
}

\begin{abstract}
Four healthy, cognitively intact elders participated. Subjects underwent fMRI scanning while performing a word recognition task with an easy condition (low demand) and a difficult condition titrated to each subject's ability (titrated demand). Relative to low, titrated demand was associated with increased activation of the left medial frontal (cluster level $P<.002$ ), right superior temporal $(P<.007)$ and right superior parietal cortices $(P<.001)$. Increased activation of bilateral cortical areas by elders during the more challenging titrated demand compared with low demand may indicate recruitment of additional brain regions, enabling subjects to maintain performance despite increasing difficulty. Alternatively, the bilateral activation on this word recognition task may reflect compensatory use of right hemisphere networks. (C) 2002 Elsevier Science Inc. All rights reserved.
\end{abstract}

Keywords: Frontal cortex; Functional magnetic resonance imaging; Normal aging; Serial word recognition

\section{Introduction}

Recognition memory declines with normal aging. Despite this decline, many elders are able to perform well on recognition memory tasks. The present study used a functional magnetic resonance imaging (fMRI) paradigm to examine which brain regions were engaged by normal elders during performance of a verbal serial recognition task. Increased activation of bilateral cortical areas by elders during the more challenging titrated demand compared with low demand was observed. This differential activation may indicate recruitment of additional brain regions, enabling subjects to maintain performance despite increasing difficulty. Other imaging studies have interpreted right-sided activation during verbal memory tasks as use of iconographic characteristics to enhance performance (Squire et al., 1992).

Abbreviations: fMRI, functional magnetic resonance imaging; ISI, interstimulis interval; PET, positron emission tomography; SPM, statistical parametric mapping; SLS, study list size

* Corresponding author. Department of Neurology, Movement Disorders, 22 South Greene Street, Baltimore, MD 21201, USA.

E-mail address: kanderson@psych.umaryland.edu (K.E. Anderson).

\section{Methods}

\subsection{Subjects}

Subjects were four normal, right-handed, female elderly volunteers, with a mean age of 76 (S.D. \pm 8.4 years). Subjects were given a medical screening examination to ensure they were healthy, and neuropsychological evaluation to rule out deficits or dementia. Informed consent was obtained from all subjects in accordance with Columbia University Institutional Review Board policy.

\subsection{Cognitive activation tasks}

Subjects performed a serial verbal memory task during imaging. This word recognition task is summarized in Table 1 . The titrate condition was a continuous performance verbal recognition test that consisted of the serial presentation of one or more single words followed by a series of recognition probes with a box around each probe, identifying it as such. For example, if the study list size (SLS) was two, two words were sequentially presented for study followed by two sequentially presented for recog- 
Table 1

Stimuli used in word recognition task

\begin{tabular}{ll}
\hline Condition & Description \\
\hline Crosshairs & Stare at fixed crosshairs for 1 min \\
Low demand & Study, then test on, one word \\
Motor control & $\begin{array}{l}\text { Alternate clicking response buttons with } \\
\text { right and left hand whenever a } \\
\text { word is on the screen } \\
\text { Titrated demand }\end{array}$ \\
& $\begin{array}{l}\text { Study, then test, on a word list determined } \\
\text { on the day prior to scanning; at } 75 \% \text { accuracy }\end{array}$ \\
\hline
\end{tabular}

nition. The subject responded with a differential button press, indicating whether each probe was or was not the same as the study word. This study-recognize sequence was repeated continuously throughout the titrate condition. The list length for the titrate condition was determined one day prior to the scan by training the subjects for two 15-min sessions on the word list learning task, using the staircase method to determine the number of words each subject identified correctly $75 \%$ of the time. This ensured that all subjects were equally challenged by the task, controlling for differences in memory ability between individual subjects. For any SLS, equal numbers of study and test items were administered during the scan period. In the low demand condition the SLS was fixed at 1 . Two other conditions were included. Fixation on crosshairs for $1 \mathrm{~min}$ (crosshairs condition) was used as baseline. Alternate clicking of response buttons with the right or left hand whenever a word came on the screen for 1 min (motor control condition) was used to separate out the effects of viewing words and pressing buttons, without a memory component.

Study items for all conditions except crosshairs were displayed visually for a 2-s duration with a $133-\mathrm{ms}$ interstimulis interval (ISI). Crosshairs were displayed over that block of the study continuously. On each trial requiring a response, offset of the last study item preceded the first test item display by $500 \mathrm{~ms}$. Test item displays were extinguished immediately following a subject's response, or after $3 \mathrm{~s}$, whichever came first. A premature response cutoff of $200 \mathrm{~ms}$ was used, with responses below this cutoff ignored. Responses automatically triggered the presentation of the next study item or test item. Fifty percent of trials required a "yes" response.

\subsection{Functional imaging}

A 1.5-T magnetic resonance scanner (General Electric Signa) retrofitted for echo planar imaging was used. An echo planar gradient echo sequence [echo time $(\mathrm{TE})=60$ $\mathrm{ms}$; repetition time $(\mathrm{TR})=3 \mathrm{~s}$; flip angle $=90^{\circ}$ ] and a standard quadrature head coil were used to acquire T2* weighted images with an inplane resolution of $3.124 \mathrm{~mm}$ $\left(64 \times 64\right.$ matrix; $20^{2} \mathrm{~cm}$ field of view). Based on a T1 saggital "scout" image, 17 contiguous, axial slices were acquired simultaneously. Slice thickness was $8 \mathrm{~mm}$. All subjects were imaged during a 12-min fMRI echo planar session. They performed each of the four components of the word recognition task twice. Stimuli were projected onto a screen which subjects viewed by looking at a prism within the head coil. Responses were recorded on a nonmagnetic handheld response device (Lightwave Medical Industries, Vancouver, BC, Canada).

\subsection{Structural imaging}

Seventeen high-resolution T2 weighted images were acquired after the fMRI procedure for corregistration into Talairach space and anatomical localization of activated regions. The total scan time was approximately $30 \mathrm{~min}$.

\subsection{Analysis of fMRI data}

Using the statistical parametric mapping (SPM96) module in Medx (Medx 3.0, Sensor Systems, Sterling, VA), functional volumes were realigned to the middle volume of each study. SPM (Friston et al., 1991) as implemented in Medx 3.0 was also used for all analyses.

Also within SPM, data were analyzed with an Analysis of Covariance (ANCOVA), and then means and difference scores were compared using specified contrasts, or "subtractions," on a pixel by pixel basis. Significant regions with cluster and/or voxel $P$ value $<.05$ were identified from the Talairach Atlas using the Talairach coordinates generated by SPM (Talairach and Tournoux, 1988). Contrasts of interest for analyses were: titrate-low demand, low demand-titrate, titrate-motor control, motor control-titrate, titrate-crosshairs, crosshairs-titrate.

\section{Results}

\subsection{Behavioral data}

The 4 elderly subjects averaged a mean SLS of 20 words (S.D. \pm 6.8 ) remembered during titrate with approximately $75 \%$ accuracy. Accuracy during low demand averaged close to $100 \%$.

\section{2. fMRI data}

fMRI results are displayed in Fig. 1 and in Table 2.

Elders demonstrated left superior frontal, occipital and superior temporal, along with right inferior frontal and parietal cortical activation during the titrate condition compared with low demand. Conversely, they showed left superior parietal, right occipital and medial frontal cortical deactivation during titrate compared with low demand. Bilateral superior occipital cortex activation was seen with titrate compared with the motor control condition; left superior parietal and lingual cortices were deactivated in 

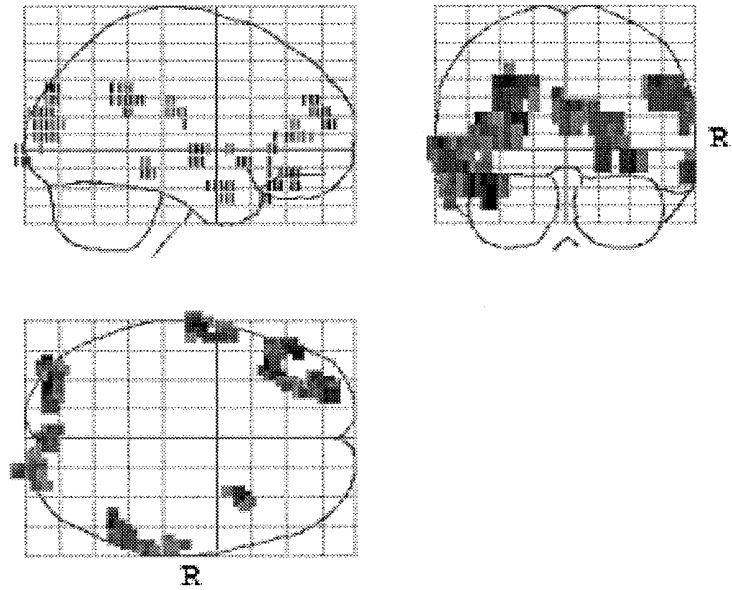

Fig. 1. Activation in four normal elders during titrated sequential memory task compared with the low demand task.

this comparison. Comparison of titrate with the crosshairs condition demonstrated increased left superior frontal and bilateral inferior frontal cortical activation, with a relative left superior parietal and right medial frontal and bilateral occipital cortex deactivation.

\subsection{Discussion}

Memory change with aging has been addressed in numerous studies, but few have examined how brain activity may change in response to a challenging test. By using a titrated memory task, this study assured that all subjects were performing at maximal level of effort, so that brain activation with optimal memory burden could be examined. Elders engaged in a sequential word recognition task differentially activated bilateral cortical regions, including frontal, temporal and parietal areas, during performance of the challenging memory task (titrate condition) compared with the easy memory task (low demand).

This increase in bilateral activation may represent greater attention and monitoring demands needed for the titrate condition, compared with the low demand condition. Such bilateral cortical increases have been reported in other fMRI studies of difficult memory judgements, such as source memory (Henson et al., 1999), and in working memory tasks that involve holding and manipulating information over time (Rypma et al., 1999). fMRI studies have also demonstrated that frontal cortex is activated when memory load is increased, even if no actual manipulation of material being maintained is required. (Rypma and D'Esposito, 1999). It is also possible that a strategy shifting occurs in subjects during the titrate condition, engaging regions typically associated with working memory tasks such as the dorsal frontal cortices. Others have reported changes in strategy to incorporate working memory domains with more difficult tasks (Deiber et al., 1991). It has been shown in prior imaging studies that tasks which are more demanding both invoke bilateral frontal activation, and also dorsal frontal activation, which was seen in the current study (D’Esposito et al., 1995; Petrides et al., 1993) with the relative left superior frontal cortical increase in activity during titrate compared with low demand. Another group (Grady et al., 1994) found that elderly subjects activated regions associated with more difficult task performance in young subjects (such as prefrontal cortex) during performance of less difficult tasks, and relative deactivation of regions engaged by young subjects, such as peristriate cortex. The authors suggested that this may be due to reduction of processing resources.

Other studies have interpreted right-sided activation during verbal memory tasks as use of iconographic characteristics of the words to improve performance as

Table 2

fMRI results from SPM analyses

\begin{tabular}{|c|c|c|c|c|c|c|}
\hline \multirow{2}{*}{$\frac{\text { Contrast }}{\text { Titrate-low demand activations }}$} & \multirow{2}{*}{$\begin{array}{l}\text { Regions } \\
\text { Left superior frontal }\end{array}$} & \multicolumn{3}{|c|}{ Talairach coord. $(\mathrm{x}, \mathrm{y}, \mathrm{z})$} & \multirow{2}{*}{$\begin{array}{l}\text { Cluster } P \\
.011\end{array}$} & \multirow{2}{*}{$\frac{\text { Voxel } P}{.001}$} \\
\hline & & -34 & 31 & -21 & & \\
\hline & Left occipital & -41 & -84 & 21 & .012 & .001 \\
\hline & Left superior temporal & -59 & -6 & 7 & .024 & .004 \\
\hline & Right inferior frontal & 41 & 19 & 0 & .046 & .012 \\
\hline & Right inferior parietal & 53 & -50 & 42 & .051 & .015 \\
\hline \multirow[t]{3}{*}{ Titrate-low demand deactivations } & Left superior parietal & -16 & -69 & -35 & .004 & .000 \\
\hline & Right occipital & 31 & -78 & 0 & .013 & .001 \\
\hline & Right medial frontal & 41 & 53 & -7 & .042 & .010 \\
\hline \multirow[t]{2}{*}{ Titrate - motor control activations } & Right superior occipital & 25 & -91 & 21 & .000 & .000 \\
\hline & Left superior occipital & -25 & -84 & 42 & .001 & .000 \\
\hline \multirow[t]{2}{*}{ Titrate-motor control deactivations } & Left superior parietal & -14 & -59 & 38 & .000 & .000 \\
\hline & Left lingual gyrus (inferior occipital) & -6 & -78 & 0 & .001 & .000 \\
\hline \multirow[t]{3}{*}{ Titrate-crosshair activations } & Left superior frontal & -22 & 62 & 21 & .003 & .000 \\
\hline & Left inferior frontal & -47 & 44 & -7 & .010 & .001 \\
\hline & Right inferior frontal & 41 & 19 & 0 & .046 & .005 \\
\hline \multirow[t]{3}{*}{ Titrate-crosshair deactivations } & Right medial frontal & 41 & 53 & -7 & .001 & .000 \\
\hline & Left lingual gyrus & -6 & -78 & 0 & .005 & .000 \\
\hline & Right inferior occipital & 31 & -84 & 0 & .011 & .001 \\
\hline
\end{tabular}


task difficulty increases (Squire et al., 1992). Prior positron emission tomography (PET) studies with this task have also shown that elders who perform better (i.e., achieve a longer list length) differentially activate anterior cortical regions, compared with those who perform less well (Stern et al., 2000). The parietal cortex activation seen in titrate may also reflect the larger memory burden of the more demanding task. Imaging studies of verbal memory span have shown posterior parietal activation (although these effects were left-sided and those in the current study were on the right) as memory load increases (Jonides et al., 1997).

During the titrate condition compared with the simple motor control task, subjects showed activation of bilateral superior occipital cortex regions. This may reflect scanning and word reading during study and test conditions, and has been seen in other PET studies when a complex task is compared to a relatively simple one (Uecker et al., 1997). In contrast, they showed deactivation of left inferior occipital and superior parietal areas in comparison to the motor task, which probably relates to motor activity such as button pressing for responses, in the absence of a significant memory burden in the motor task. As would be expected, subjects demonstrated frontal cortex activation during the complex titrate condition compared with the simple crosshairs baseline, which had neither memory demands nor motor requirements.

Activation of bilateral cortical areas by elders during the more challenging titrate condition compared with low demand may indicate recruitment of additional neuronal resources, enabling subjects to maintain performance despite a heavy memory burden. The small number of subjects in this study may also contribute to variations in the results, and could be the reason some expected activations were not seen. Bilateral activation may reflect compensatory use of right hemisphere networks. The strong propensity toward frontal cortex activation during the titrate condition may aid in mediation of organizational task demands.

\section{Conclusion}

Normal elders demonstrated left superior frontal, occipital and superior temporal, along with right inferior frontal and parietal cortical activation during a challenging titrated sequential word recognition condition compared with a relatively trivial word recognition task. Conversely, they showed left superior parietal, right occipital and medial frontal cortical deactivation on the titrated task compared with the easy one. Other conditions used in the study to control for motor elements of the task and word viewing elicited relative posterior activation compared with the frontal recruitment seen in the titrated memory condition. The increased cortical activation seen in the demanding titrated task may represent attempts by the elders to cope with heavy memory demands of the task, along with task processing strategies.

\section{References}

D’Esposito, M., Detre, J., Alsop, D., Shin, R., Atlas, S., Grossman, M., 1995. The neural basis of the central executive system of working memory. Nature 378, 279-281.

Deiber, M.P., Passingham, R.E., Friston, K.J., Nixon, P.D., Frackowiak, R.S., 1991. Cortical areas and the selection of movement: a study with positron emission tomography. Exp. Brain Res. 84, 393-402.

Friston, K.J., Frith, C.D., Liddle, P., Frackowiak, R.S.J., 1991. Comparing functional (PET) images: the assessment of significant change. J. Cereb. Blood Flow Metab. 11, 690-699.

Grady, C.L., Maisog, J.M., Horwitz, B., Ungerleider, L.G., Mentis, M.J., Salerno, J.A., Pietrini, P., Wagner, E., Haxby, J.V., 1994. Age-related changes in cortical blood flow activation during visual processing of faces and location. J. Neurosci. 14 (3), 1245-1462.

Henson, R.N., Shallice, T., Dolan, R.J., 1999. Right prefrontal cortex and episodic memory retrieval: a functional MRI test of the monitoring hypothesis. Brain 122 (7), 1367-1381.

Jonides, J., Schumacher, E.H., Smith, E.E., Lauber, E.J., Awh, E., Minoshima, S., Koeppe, R.A., 1997. J. Cognit. Neurosci. 9, 462-475.

Petrides, M., Alivisatos, B., Meyer, E., Evans, A., 1993. Functional activation of the human prefrontal cortex during the performance of verbal working memory tasks. Proc. Natl. Acad. Sci. USA 90, 878-882.

Rypma, B., D'Esposito, M., 1999. The roles of prefrontal brain regions in components of working memory: effects of memory load and individual differences. Proc. Natl. Acad. Sci. USA 96, 6558-6563.

Rypma, B., Prabhakaran, V., Desmond, J.E., Glover, G.H., Gabrieli, J.D., 1999. Load dependent roles of frontal brain regions in the maintenance of working memory. NeuroImage 9, 216-226.

Squire, L.R., Ojemann, J.G., Miezin, F.M., Petersen, S.E., Videen, T.O., Raichle, M.E., 1992. Activation of the hippocampus in normal humans: a functional anatomical study of memory. Proc. Natl. Acad. Sci. USA 89 (5), 1837-1841.

Stern, Y., Moeller, J.R., Anderson, K.E., Luber, B., Zubin, N.R., DiMauro, A., Park, A., Campbell, C.E., Marder, K., Bell, K., Van Heertum, R., Sackeim, H.A., 2000. Different brain networks mediate task performance in normal aging and AD. Neurology 55, 1291-1297.

Talairach, J., Tournoux, P., 1988. Co-planar stereotaxic atlas of the human brain. Theime Medical Publishers, New York, pp. 40-110.

Uecker, A., Reiman, E.M., Schacter, D.L., Polster, M.R., Cooper, L.A., Yun, L.S., Chen, K., 1997. Neuroanatomical correlates of implicit and explicit memory for structurally possible and impossible visual objects. Learn. Mem. 4, 337-355. 